International Journal of Engineering, Science and Technology Vol. 9, No. 1, 2017, pp. 55-68
INTERNATIONAL JOURNAL OF ENGINEERING, SCIENCE AND TECHNOLOGY

www.ijest-ng.com

www.ajol.info/index.php/ijest

(C) 2017 MultiCraft Limited. All rights reserved

\title{
Solution of optimal power flow using evolutionary-based algorithms
}

\author{
Amr K. Khamees ${ }^{1}$, Ahmed El-Rafei ${ }^{1}$, N. M. Badra ${ }^{1}$, Almoataz Y. Abdelaziz $^{2} *$ \\ ${ }^{I}$ Department of Engineering Physics and Mathematics, Faculty of Engineering, Ain Shams University, Cairo, EGYPT \\ ${ }^{2}$ Department of Electrical Power \& Machines, Faculty of Engineering, Ain Shams University, Cairo, EGYPT \\ *Corresponding Author: e-mail: almoatazabdelaziz@hotmail.com,almoataz_abdelaziz@eng.asu.edu.eg
}

\begin{abstract}
This paper applies two reliable and efficient evolutionary-based methods named Shuffled Frog Leaping Algorithm (SFLA) and Grey Wolf Optimizer (GWO) to solve Optimal Power Flow (OPF) problem. OPF is one of the essential functions of electrical power generation control and operation. It aims to estimate the optimal settings of real generator output power, bus voltage, reactive power compensation devices, and transformer tap setting. The objective function of OPF is to minimize total production cost while maintaining the power system operation within its security limit constrains. SFLA and GWO are meta-heuristic evolutionary-based methods. SFLA mimics the frogs' behavior while searching for food. GWO mimics the grey wolfs behavior while hunting for prey. These methods are simulated and tested on the standard IEEE 30-bus and 57-bus test systems. Moreover, the performances of the applied algorithms are analyzed and compared with each other as well as with other existing optimization techniques. The obtained results show the strengths of the two applied methods and the superiority of them in comparison to the other methods.
\end{abstract}

Keywords: Optimal power flow, Shuffled Frog Leaping, Memeplex, Grey Wolf Optimizer.

DOI: http://dx.doi.org/10.4314/ijest.v9i1.5

\section{Introduction}

Optimal power flow is a load flow analysis that uses optimization methods to adjust decision variables (control variables) to determine the best operating conditions of the power system. The control variables of OPF are the generator active power output, voltage of generating unit, tap-settings of the transformers, and shunt reactive power of injection devices in the electrical power system. The objective function of OPF aims to minimize the total generation cost while satisfying the equality and inequality constrains (Al-Muhawesh and Qamber, 2008; Vaisakh and Srinivas, 2010). The equality constraints are the active and reactive power balance at each node. The inequality constraints are the control variables limits, line flow limits, and system voltage limits. The OPF was developed in 1960s as an extension to economic load dispatch (ELD) (Kirschen and Strbac, 2004).

Mathematically, OPF is a non-linear large-scale optimization problem subject to non-linear constrains. Various traditional techniques have been employed to solve the OPF problem since 1960s. Among these are the following classical methods: Linear programming-based algorithms (LP) (Lin et al., 2010; Momoh et al., 1999) which need linearization for the objective function as well as constraints. Non-linear optimization-based methods (NLP) (Momoh et al., 1999) are used in case of nonlinear objective function or constrains. Newton methods (Ambriz-Perez et al., 2000; Ambriz-Perez et al., 1998) are second order optimization techniques which are based on Taylor series. Quadratic programming approach (QP) (Momoh et al., 1994) is a type of NLP that is used when the objective function is quadratic and the constraints are linear. Gradient based methods (Dommel and Tinney, 1968) depend on moving along the direction that improves the objective function as determined by the gradient vector. Decomposition algorithms (Alsac and Stott, 1974; Shoults and Sun, 1982) divide the optimization task by partitioning the constrain limits into smaller constrained problems. 
All the aforementioned techniques and other traditional methods have been applied to OPF. In addition, many improvements have been introduced to classical techniques (Alguacil and Conejo, 2000; Wei et al., 1998). However, they generally suffer from many drawbacks in solving complex problems like OPF optimization. The main disadvantages can be summarized as follows:

- Some techniques require linearization and differentiability.

- The obtained optimal solution is dependent on initialization thus, the optimization could end at local optima and misses the global optimum solution.

- Some techniques suffer from poor convergence.

- The convergence becomes slow in case of large number of control variables.

Due to the drawbacks in classical methods, the artificial intelligence (AI) techniques have been introduced to solve the OPF problem. The AI-based optimization has become an important approach for determining the global optimal solution. One of the most important intelligent search techniques is called evolutionary algorithms (EAs). Since many EAs have natural or biological origins, they are also referred to as natural inspired (NI) or bio-inspired optimization (BIO) algorithms. EAs generate a random population that includes a set of possible solutions. The populations carry out a comprehensive search in the entire problem's search space to obtain the global optimal solution. This procedure of searching leads to reduction in execution time, enhancing solution quality, and avoids local optimum solutions. The main advantages of EAs include their ability of finding the global optimal solution, processing problems having many qualitative constraints, better than classical methods in solving multi-objective optimization problems, and the possibility of simultaneously locating multiple local optimal solutions.

A literature survey shows that various EAs optimization techniques have been employed to solve OPF problem during the past few decades. The following methods are examples of EAs:

Genetic algorithm (GA) (Zaman et al., 2016) operates with encoded binary parameters rather than the decimal values. Each string considered as a chromosome which presents all possible solutions. This method depends on three main operators which are selection, crossover, and mutation.

The evolutionary algorithm (Toffolo and Lazzaretto, 2002) depends on operators inspired by biological evolution like procreation, crossover, mutation, and selection.

Tabu search (TS) (Senthil and Manikandan, 2010) is a meta-heuristic search method that employs local search algorithms based on a hill-climbing technique. This method utilizes local search steps to move from a local solution to an improved global optimal solution by defining neighborhoods and using tabu list.

Particle swarm optimization (PSO) (Praveen and Rao, 2016) is a stochastic optimization technique. PSO is inspired by the procedure followed by organized colonies like fish or birds when searching for food.

Artificial neural network (ANN) (Suman et al., 2016) is a connecting group of artificial neurons which uses a computational paradigm for data processing based on connectionist models.

Artificial bee colony optimization (ABC) (Afandi and Miyauchi, 2014) simulates foraging attitude of a bee swarm during searching for a food source and sharing information between bees.

Differential Evolution (DE) (Acharjee, 2016) is a population-based stochastic optimization technique. The initial population is generated randomly and then moves to the optimal solution using a combination of natural arithmetic and evolutionary operators like recombination, mutation, and selection.

Naturally inspired algorithms are also used to solve the ELD problem. A comprehensive review of application of BIO optimization algorithms to the ELD problem is presented in (Dubey et al., 2014). Among the recent ones is the Flower Pollination Algorithm (FPA) (Dubey et al., 2015) and Backtracking search algorithm (BSA) (Tyagi et al., 2016). FPA is an intriguing process in the natural world. The local pollination of FPA is controlled by a scaling factor and an intensive exploitation phase is added to tune the best solution. BSA is a population based stochastic search optimization technique which is based on the principles of evolution and natural genetics by using mutation, crossover, and selection.

Shuffled frog leaping algorithm (SFLA) was proposed by Eusuff and Lansey in 2003 (Eusuff and Lansey, 2003). It is a memetic algorithm that is based on the evolution of memes where the population (frogs) performs a local optimization inside their memeplex. The frogs within each memeplex are improved independently. The frogs spread their memes (idea) through the memeplexes. SFLA has a local search technique and a global search that depends on mixing data between memeplexes to obtain a global optimal solution. The SFLA has fast execution time as a small population of frogs is sufficient to obtain a convergence to the global optimum solution.

A recent evolutionary-based technique is the grey wolf optimizer (GWO) that was proposed by Mirjalili in 2014 (Mirjalili et al., 2014). The GWO simulates the hunting and leadership hierarchy of grey wolves. It depends on the steps taken by the wolves for hunting the prey. The grey wolves are divided into four types according to their fitness in leadership hierarchy. The optimization procedure contains three main steps which are searching for prey, encircling prey, and attacking prey.

This paper applies the two reliable and efficient evolutionary-based methods, SFLA and GWO, for solving the OPF problem. The standard IEEE 30-bus and 57-bus are used as test systems. Moreover, the performance of these algorithms is analyzed and compared to other state of the art techniques to show the strengths and suitability of each method in solving the OPF problem. 


\section{The Formulation of OPF Problem}

The goal of OPF is to obtain the optimal steady state power system setting to minimize an objective function such as generation cost while the power system is operating within its security limit. The objective function $\mathrm{F}_{\mathrm{T}}$ is the generator fuel cost which is given by the following equation

$$
F_{T}=\sum_{i=1}^{N g} a_{i} P_{i}^{2}+b_{i} P_{i}+c_{i} \quad \$ / \mathrm{hr}
$$

where,

$\mathrm{F}_{\mathrm{T}} \quad$ : Total quadratic cost function

$P_{\mathrm{i}} \quad$ : Real power generated

$\mathrm{Ng}$ : Number of generator bus

$a_{i}, b_{i}, c_{i}:$ Fuel cost coefficients

\section{-Subject to the constraints}

(a) Network real power balance

$P_{i}(V, \delta)-P_{g i}+P_{d i}=0$

$$
(\mathrm{i}=1,2, \ldots, \mathrm{NB})
$$

(b) Network reactive power balance

$Q_{i}(V, \delta)-Q_{g i}+Q_{d i}=0$

$$
(\mathrm{i}=\mathrm{NV}+1, \mathrm{NV}+2, \ldots, \mathrm{NB})
$$

(c) Security limits constraints

- Generator active power constrains

$P_{i, \text { min }}^{g} \leq P_{i}^{g} \leq P_{i, \text { max }}^{g}$

$$
(\mathrm{i}=1,2, \ldots, \mathrm{NG})
$$

- Generator voltage magnitude constrains

$V_{i, \text { min }}^{g} \leq V_{i}^{g} \leq V_{i, \text { max }}^{g}$

$$
(\mathrm{i}=1,2, \ldots, \mathrm{NG})
$$

- Voltage angle constrains

$\delta_{i, \min } \leq \delta_{i} \leq \delta_{i, \max }$

$$
(i=1,2, \ldots, N B)
$$

- Real active power flow equations are:

$P_{i}(V, \delta)=V_{i} \sum_{j=1}^{N B} V_{j}\left(G_{i j} \cos \delta_{i j}+B_{i j} \sin \delta_{i j}\right)$

- Reactive power flow equations are:

$Q_{i}(V, \delta)=V_{i} \sum_{j=1}^{N B} V_{j}\left(G_{i j} \sin \delta_{i j}-B_{i j} \cos \delta_{i j}\right)$

where, 
NG : Total number of generator buses

NB : Total number of number of buses

$\mathrm{NV}$ : Total number of voltage controlled buses

$\mathrm{P}_{\mathrm{i}}, \mathrm{Q}_{\mathrm{i}}$ : Active and reactive power injection into bus $\mathrm{i}$

$\mathrm{P}_{\mathrm{di}}, \mathrm{Q}_{\mathrm{di}}$ : Active and reactive load on bus $\mathrm{i}$

$\mathrm{P}_{\mathrm{gi}}, \mathrm{Q}_{\mathrm{gi}}$ : Active and reactive power generation on bus $\mathrm{i}$

$\mathrm{V}_{\mathrm{i}}$ : Voltage magnitude on bus $\mathrm{i}$

$\delta_{i}$ : Voltage phase angle on bus $\mathrm{i}$

$\mathrm{Y}_{\mathrm{ij}}=\mathrm{G}_{\mathrm{ij}}+\mathrm{jB}_{\mathrm{ij}}$ : Admittance matrix elements

\section{Shuffled Frog Leaping Algorithm}

The basic idea behind SFLA is inspired from the frogs' memetic evolution while searching for food. The two main components of the SFLA are the local and global search. The local search is similar to the one used in PSO algorithm (Eberhart and Kennedy, 1995). The global search is similar to the method used in shuffled complex evolution algorithm (Duan et al., 1992). The solution of problem is generated as a random population (frogs) as shown in Equation (1). Each raw is considered as one frog which represents one possible solution for the problem. The frogs are divided into sets "memeplexes". Each memeplex has the same number of frogs. Within each memeplex, there is a local search where the frogs improve their positions to get a better solution. In the global information exchange technique, the frogs are divided again into memeplexes based on their fitness. Therefore, the data are passed across memeplexes in a shuffling process (Eusuff and Lansey, 2003).

The procedure of SFLA is as follows:

1) Generate initial population of $P$ frogs which represents the randomly generated solution

Population $=\left[\begin{array}{c}X_{1} \\ \cdot \\ \cdot \\ X_{p}\end{array}\right]_{P \times N_{\text {tie }}}$

where $\mathrm{X}_{\mathrm{i}}$ is the position of the $\mathrm{i}^{\text {th }}$ frog, $\mathrm{P}$ is the number of frogs, and $\mathrm{N}_{\text {tie }}$ is the problem dimension

2) The frogs are arranged in a descending order according to their fitness.

3) Frogs are divided into $m$ memeplexes, each memeplex contains $n$ frogs (i.e., $P=n \times m$ ) where the $1^{\text {st }}$ frog of the sorted frogs is inserted into the $1^{\text {st }}$ memeplex, the $2^{\text {nd }}$ is added to the $2^{\text {nd }}$ memeplex, and so on till the $\mathrm{m}^{\text {th }}$ frog is inserted into $\mathrm{m}^{\text {th }}$ memeplex, then $(\mathrm{m}+1)^{\text {th }}$ frog is concatenated to the $1^{\text {st }}$ memeplex again, and so on. Therefore, each memeplex has $\mathrm{n}$ frogs

4) Within each memeplex, there is a local search where the best frog $X_{b}$, worst frog $X_{w}$, and global best frog $X_{g}$ are identified. Then, the worst frog position is modified using frog leaping rule as given by Equation 10 and Equation 11 as indicated in Figure 1:

$$
\begin{aligned}
& \mathrm{D}_{\mathrm{i}}=\operatorname{rand} \times\left(\mathrm{X}_{\mathrm{b}}-\mathrm{X}_{\mathrm{w}}\right) \\
& \mathrm{X}_{\mathrm{w}}(\text { new })=\mathrm{X}_{\mathrm{w}}(\text { old })+\mathrm{D}_{\mathrm{i}}
\end{aligned}
$$$$
\left(-\mathrm{D}_{\min } \leq \mathrm{D}_{\mathrm{i}} \leq \mathrm{D}_{\max }\right)
$$

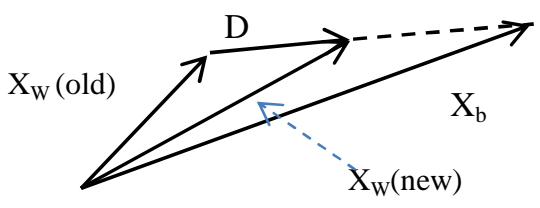

Figure 1: Frog leaping rule 
where rand is a random value between 0 and $1, D$ is the change frog's position, $i$ is iteration number, and $D_{\min }$ and $D_{\max }$ are the allowable minimum and maximum change in the position of frog, respectively. If $\mathrm{X}_{\mathrm{w}}(\mathrm{new})$ is better than $\mathrm{X}_{\mathrm{w}}(\mathrm{old})$, it will replace its position, otherwise, the calculations in the previous Equations are repeated with respect to the frog with the global solution. If no improvement becomes possible, then new frogs are generated randomly to replace the worst frogs.

5) Repeat the preceding step for a predetermined number of local search iterations

6) After updating the frog's position, the frogs are arranged again in a descending order by their fitness.

7) If the maximum number of iteration is reached, then stop. Else, return back to step 3.

The flowchart of SFLA is shown in Figure 2a and 2b.

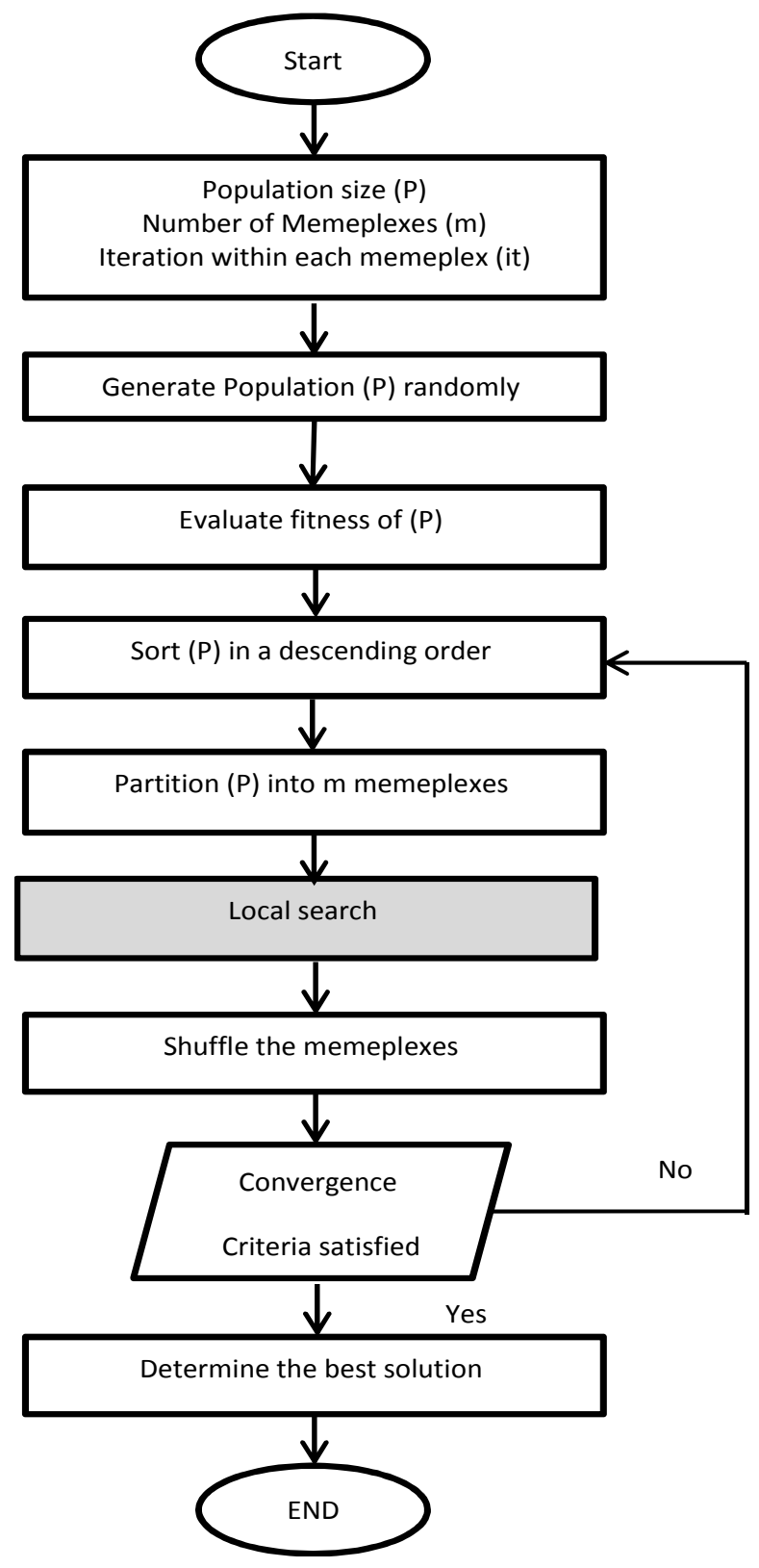

Figure 2a: Flowchart of SFLA 


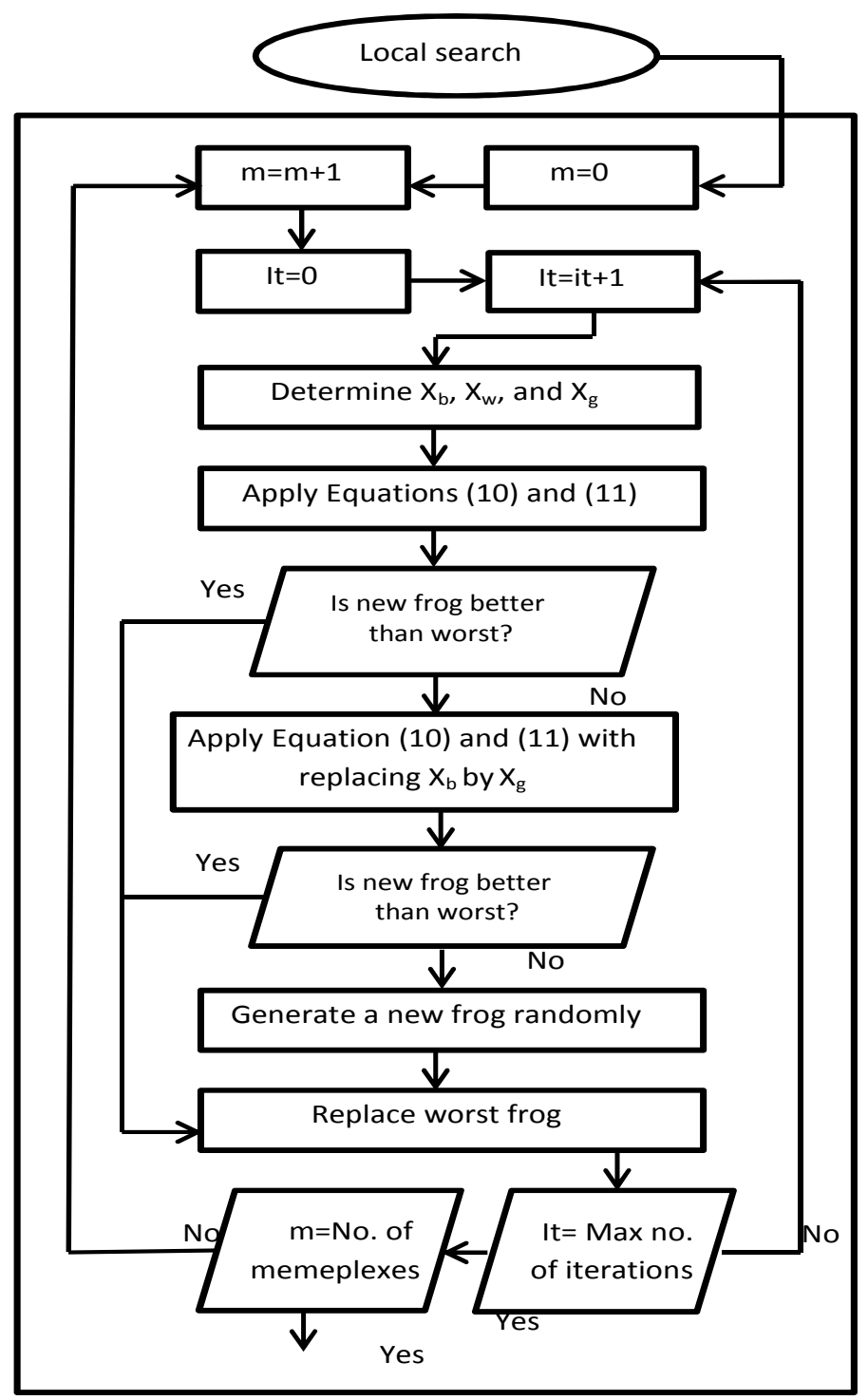

Figure 2b: Flowchart of SFLA Local search

\section{Grey Wolf Optimizer}

Grey wolf optimizer is a metaheuristic search approach that is inspired by the hunting process followed by wolves. The wolves always prefer to live in groups each having 5-12 wolves. Wolves are categorized into four dominant positions which are (1) alpha $(\alpha),(2)$ beta $(\beta),(3)$ delta $(\delta)$, and (4) omega $(\omega)$ wolves as shown in Figure 3 (Mirjalili et al., 2014).

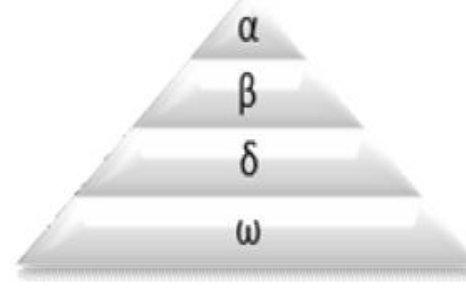

Figure 3. Hierarchy of grey wolves

The first predominant wolves are alpha wolves and the predominance decreases gradually till the omega wolves. The alpha wolves take the leading decisions like walk, hunt, and sleep decisions. Also, alpha wolves could follow other wolves. Although, 
alpha wolves have the highest dominance but sometimes it's not the strongest wolves in the group (Mech, 1999). The beta wolves are the second predominance level and they help alpha in some leadership activities. They are appointed to alpha wolves, if alpha wolves become very old or endear. The beta wolves are consultants of the alpha wolves and also leaders for the delta and omega wolves. The omega wolves are the lowest predominance in wolves group. The delta wolves are predominant to omega wolves and also present data to alpha and beta wolves. The procedure of the GWO is demonstrated in Figure 4 and summarized by the following steps:

\section{a) Social strategy}

The alpha wolf is considered the best solution. Then, the beta and delta wolves are the 2nd and 3rd fittest solutions, respectively. The omega wolves are the remainder of the solutions. In general, alpha, beta, and delta wolves lead the optimization operation and omega wolves follow them.

b) Encircling the prey

The wolves are encircling the prey during hunting. In this step, the wolves move towards the prey. The encircling operation is described by the following equations:

$$
\begin{aligned}
& \vec{D}=\left|\vec{C} \bullet \vec{X}_{P}(t)-\vec{X}(t)\right| \\
& \vec{X}(t+1)=\vec{X}_{P}(t)-\vec{A} \bullet \vec{D}
\end{aligned}
$$

where $\mathrm{t}$ is current iteration number, $\vec{A}$ and $\vec{C}$ are the coefficient vectors, $\vec{X}_{P}$ is the prey position vector and $\vec{X}$ is the search agent position vector.

The vectors $\vec{A}$ and $\vec{C}$ are calculated as follows:

$$
\vec{A}=2 \vec{a} \bullet \overrightarrow{r_{1}}-\vec{a}
$$

$\vec{C}=2 \bullet \vec{r}_{2}$

where $\vec{r}_{1}$ and $\vec{r}_{2}$ are random vectors from 0 to 1 and $\vec{a}$ is a vector that is linearly decreases from 2 to 0 over the iteration.

\section{c) Hunting}

To model the wolves' hunting procedure, it is assumed that alpha (best solution), beta, and delta wolves have adequate information for prey position. Thus, the best three solutions are saved and utilized to modify their positions. The wolves' positions are updated using alpha, beta, and delta positions to hunt the prey. The hunting operation is described by the following equations.

$$
\begin{aligned}
& D_{\alpha}=\left|C_{1} \bullet X_{\alpha}-X\right|, D_{\beta}=\left|C_{2} \bullet X_{\beta}-X\right| \\
& D_{\delta}=\left|C_{3} \bullet X_{\delta}-X\right| \\
& X_{1}=X_{\alpha}-A_{1} \bullet D_{\alpha}, X_{2}=X_{\beta}-A_{2} \bullet D_{\beta} \\
& , X_{3}=X_{\delta}-A_{3} \bullet D_{\delta} \\
& X(t+1)=\frac{X_{1}+X_{2}+X_{3}}{3}
\end{aligned}
$$

where $X_{\delta}, X_{\beta}$, and $X_{\delta}$ are positions of alpha, beta, and delta wolves respectively and A, C are vectors calculated by Equation (14) and Equation (15)

\section{d) Attacking the prey}

Wolves start attacking the prey after they stop moving. This can be expressed by decreasing the value of $\vec{a}$ from 2 to 0 iteratively. When vector $\vec{A}<1$, the wolves is forced to attack the prey (local search of GWO). 
The wolves search for the prey based on the alpha, beta, and delta positions. The wolves splay from each other for searching and then gather to attack. The prey is found when $|\mathrm{A}|>1$. The wolves are forced to search for the best prey (global search of GWO).

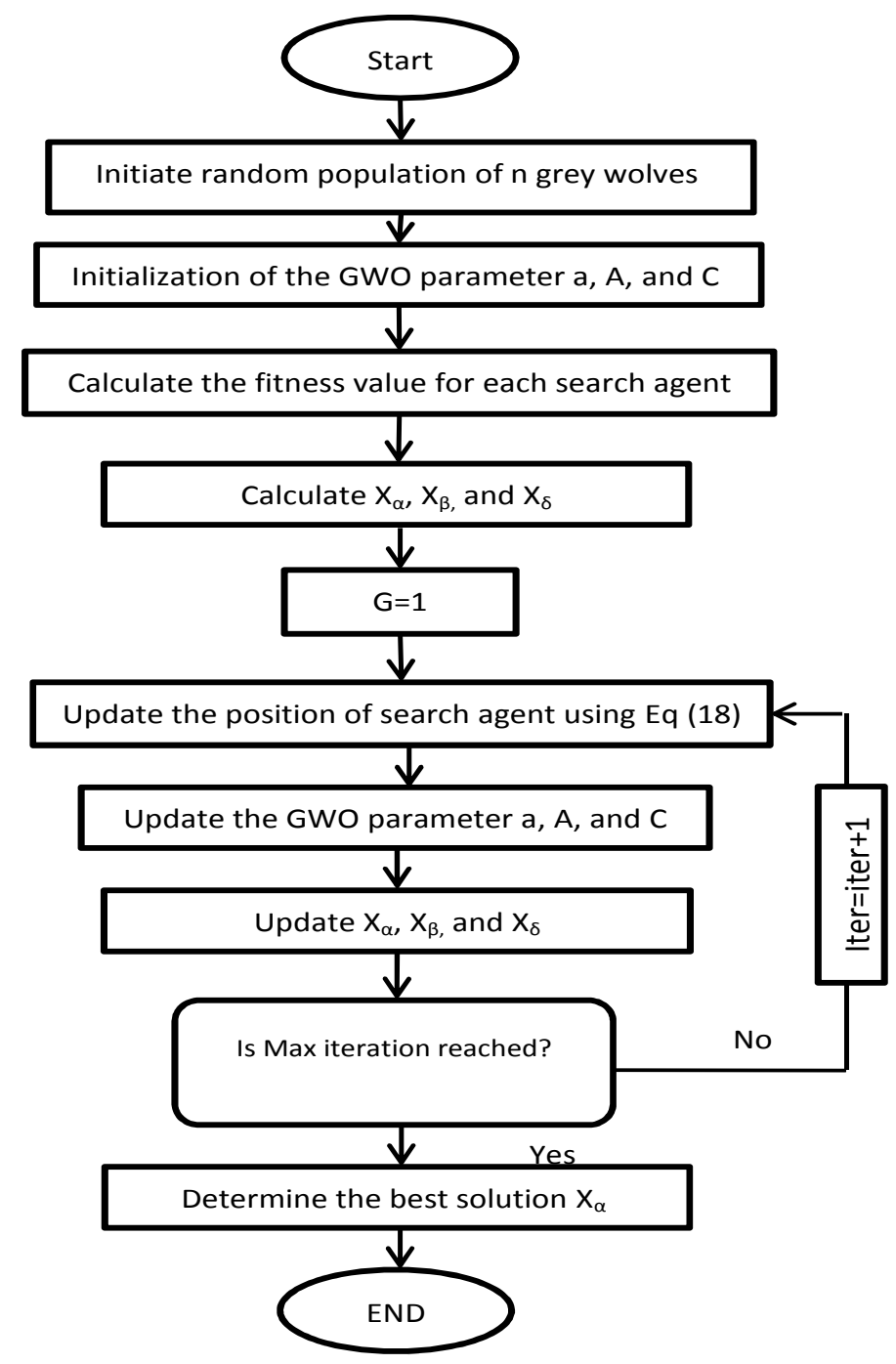

Figure 4. GWO algorithm

\section{Simulation Results}

The SFLA and GWO methods are applied to the standard IEEE 30-bus and 57-bus test systems. The generator coefficients and output generation power limits are indicated in Table 1 and Table 2. The proposed algorithms are coded in MATLAB software (Mathworks, Inc., Natick, MA, USA). This paper uses maximum number of iterations as stopping criteria in both methods. The Newton-Raphson method used for power flow solution for test systems is obtained from Matpower (Zimmerman et al., 1997)..

Table 1. Generators' coefficients for IEEE 30-bus network

\begin{tabular}{|c|c|c|c|c|c|}
\hline Bus no. & $\mathrm{a}$ & $\mathrm{b}$ & $\mathrm{c}$ & $\mathrm{P} \min$ & $\mathrm{P} \max$ \\
\hline 1 & 0.00375 & 2 & 0 & 50 & 200 \\
\hline 2 & 0.0175 & 1.75 & 0 & 20 & 80 \\
\hline 5 & 0.0625 & 1 & 0 & 15 & 50 \\
\hline
\end{tabular}


Table 1 (cont'd). Generators' coefficients for IEEE 30-bus network

\begin{tabular}{|c|c|c|c|c|c|}
\hline Bus no. & $\mathrm{a}$ & $\mathrm{b}$ & $\mathrm{c}$ & $\mathrm{P} \min$ & $\mathrm{P} \max$ \\
\hline 8 & 0.0083 & 3.25 & 0 & 10 & 35 \\
\hline 11 & 0.025 & 3 & 0 & 10 & 30 \\
\hline 13 & 0.025 & 3 & 0 & 12 & 40 \\
\hline
\end{tabular}

Table 2. Generators' coefficients for IEEE 57-bus network

\begin{tabular}{|c|c|c|c|c|c|}
\hline Bus no. & $\mathrm{a}$ & $\mathrm{b}$ & $\mathrm{c}$ & $\mathrm{P} \min$ & $\mathrm{P} \max$ \\
\hline 1 & 0.07758 & 20 & 0 & 0 & 575.88 \\
\hline 2 & 0.01 & 40 & 0 & 0 & 100 \\
\hline 3 & 0.25 & 20 & 0 & 0 & 140 \\
\hline 6 & 0.01 & 40 & 0 & 0 & 100 \\
\hline 8 & 0.022222 & 20 & 0 & 0 & 550 \\
\hline 9 & 0.01 & 40 & 0 & 0 & 100 \\
\hline 12 & 0.032258 & 20 & 0 & 0 & 410 \\
\hline
\end{tabular}

The optimal setting of control variables after applying the proposed SFLA and GWO for the standard IEEE 30-bus and 57-bus test systems are shown in Table 3 and Table 4, respectively.

Table 3. Optimal setting of control variable(s) for IEEE 30-bus system

\begin{tabular}{|c|c|c|}
\hline & SFLA & GWO \\
\hline PG1 (MW) & 177.1036 & 176.8493 \\
\hline PG2 (MW) & 48.9199 & 48.7931 \\
\hline PG5 (MW) & 21.5012 & 21.5169 \\
\hline PG8 (MW) & 21.8577 & 21.6015 \\
\hline PG11 (MW) & 12.1606 & 11.6872 \\
\hline PG13 (MW) & 11.2553 & 12.3393 \\
\hline V1 (V P.U) & 1.0600 & 1.0600 \\
\hline V2 (V P.U) & 1.0430 & 1.0430 \\
\hline V5 (V P.U) & 1.0100 & 1.0100 \\
\hline V8 (V.U) & 1.0100 & 1.0100 \\
\hline V11 (V P.U) & 1.0820 & 1.0820 \\
\hline V13 (V P.U) & 1.0710 & 1.0710 \\
\hline Losses (MW) & 9.3982 & 9.3572 \\
\hline Fuel cost (\$/h) & 801.8280 & 801.8652 \\
\hline
\end{tabular}

Table 4. Optimal setting of control variable(s) for IEEE-57 bus system

\begin{tabular}{|c|c|c|}
\hline & SFLA & GWO \\
\hline PG1 (MW) & 144.856 & 145.42 \\
\hline PG2 (MW) & 93.0378 & 95.66 \\
\hline PG3 (MW) & 45.209 & 45.02 \\
\hline PG6 (MW) & 68.2624 & 67.57 \\
\hline PG8 (MW) & 457.0264 & 454.28 \\
\hline PG9 (MW) & 95.8565 & 94.11 \\
\hline PG12 (MW) & 365.9573 & 367.95 \\
\hline V1 (V P.U) & 1.04 & 1.04 \\
\hline V2 (V P.U) & 1.01 & 1.01 \\
\hline V3 (V P.U) & 0.985 & 0.985 \\
\hline V6 (V P.U) & 0.98 & 0.98 \\
\hline V8 (V P.U) & 1.005 & 1.005 \\
\hline V9 (V P.U) & 0.98 & 0.98 \\
\hline V12 (V P.U) & 1.015 & 1.015 \\
\hline Fuel cost (\$/h) & 41872.9 & 41873.188 \\
\hline
\end{tabular}


In this work, the performance of SFLA, GWO, and GA (MATLAB GA-toolbox) are compared for solving the OPF problem on the standard IEEE 30-bus and 57-bus test systems. Table 5 compares the number of parameters, execution time, and optimal fuel cost for SFLA, GWO, and GA. The results show that the optimal fuel cost of GWO and SFLA are almost the same and better than GA. GWO has the lowest execution time and GA is faster than SFLA. GWO has only two parameters to adjust which are the population size and vector $\vec{a}$. SFLA has 5 parameters which are the number of memeplexes, number of frogs in memeplex, number of frogs in a sub memeplex, number of evolution, and maximum step size. GA has 6 parameters which are population size, number of generations, crossover type, crossover rate, mutation types, and mutation rate. These results demonstrate the superiority of the SFLA and GWO over GA method.

The performance of proposed methods is sensitive to the parameters used, Table 6 shows the values of the parameters used for each algorithm to solve the optimal power flow problem. The parameters' values were determined to obtain the optimum solution with minimal execution time. For the GWO, the optimal value for population size is 30 candidates. Using fewer candidates lead to reduction in the quality of solution while having more candidates resulted in increasing the execution time with no noticeable improvement in solution. Moreover, the solution is found to be insensitive to the vector a. For SFLA, the number of memeplexes, frogs per memeplex, frogs per sub memeplex, and number of local search iterations in SFLA are selected by a grid search to obtain the optimum solution and the lowest execution time. Using more frogs and a higher number of local iterations leads to increase in execution time. Solution is found to be insensitive to step size. The same parameters' selection criteria were also applied to GA.

Figure 5 and Figure 6 show the convergence characteristic of the proposed SFLA and GWO methods in comparison with the GA approach. As evident from the graphs, the two applied methods converge to the optimal solution after fewer number of iterations than the GA.

Table 5. Result comparison for GWO, SFLA, and GA

\begin{tabular}{|c|c|c|c|}
\hline & GWO & SFLA & GA \\
\hline Optimal cost for (30 bus) $(\$ / \mathrm{h})$ & 801.8652 & 801.8280 & 802.5 \\
\hline Optimal cost for $(57$ bus) $(\$ / \mathrm{h})$ & 41873.19 & 41872.9 & 42128 \\
\hline execution time for (30 bus) Sec & 86.384 & 214.284 & 188.198 \\
\hline execution time for (57 bus) Sec & 229.365 & 987.806 & 401.813 \\
\hline No. of parameters & 2 & 5 & 6 \\
\hline
\end{tabular}

Table 6. Selection of parameter

\begin{tabular}{|c|c|}
\hline \multicolumn{2}{|c|}{ GWO } \\
\hline Population size & 30 \\
\hline vector a & vector linearly decreases [2 to 0] \\
\hline number of memeplexes & 5 \\
\hline frogs per memeplex & 10 \\
\hline frogs per sub memeplex & 3 \\
\hline number of local search iteration & 5 \\
\hline and maximum step size & 2 \\
\hline Population size & 30 \\
\hline number of generations & 50 \\
\hline crossover type & single point \\
\hline Crossover rate & 0.9 \\
\hline mutation types & uniform \\
\hline mutation rate & 0.01 \\
\hline
\end{tabular}




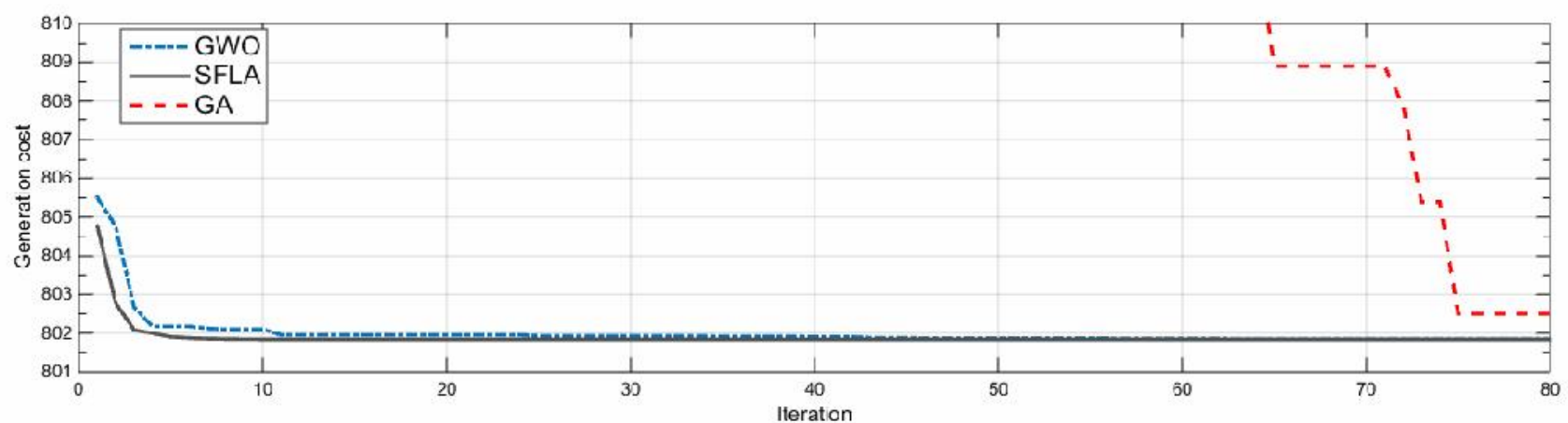

Figure 5: Convergence characteristic for GWO, SFLA and GA for the IEEE 30-bus system.

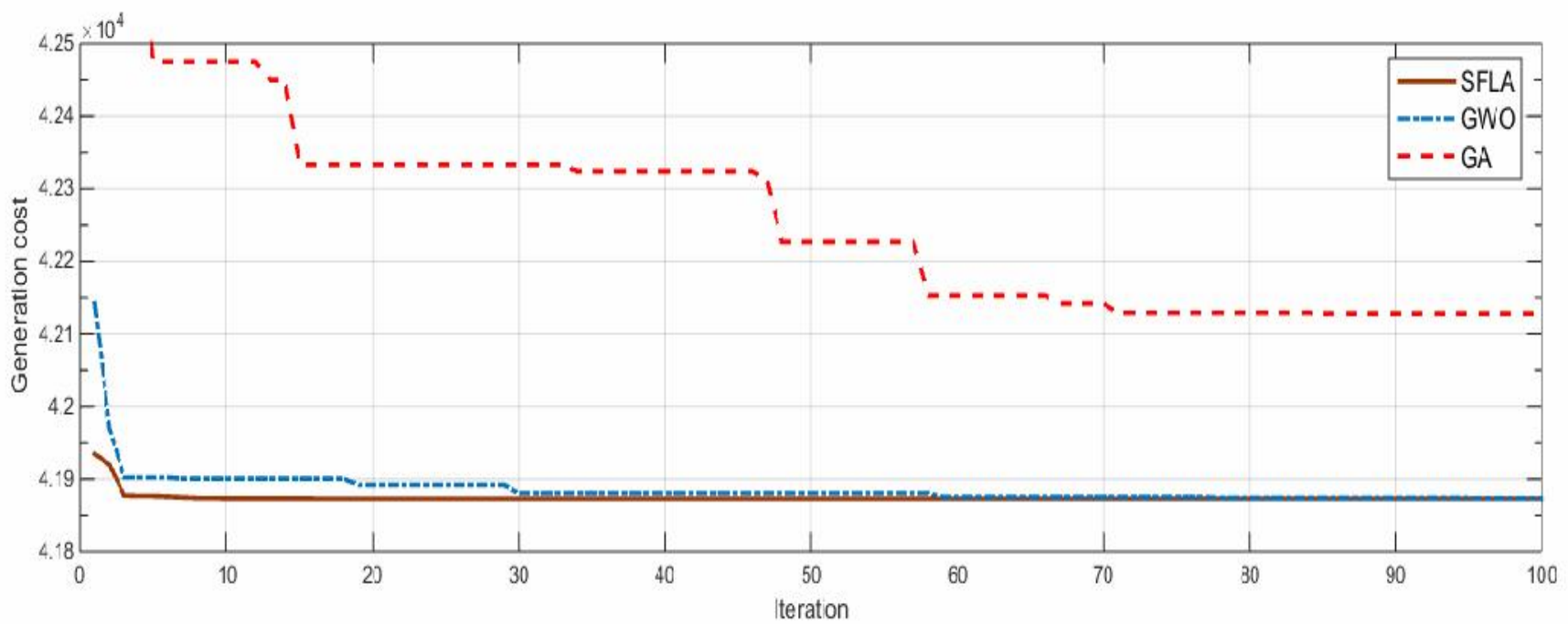

Figure 6: Convergence characteristic for GWO, SFLA and GA for the IEEE 57-bus system.

Also, SFLA and GWO are compared with EP (Yuryevich and Wong, 1999), DE (Sayah and Zehar, 2008), GA (Bouzeboudja et al., 2005; Kumar and Chaturvedi, 2013), PSO (Chalermchaiarbha and Ongsakul, 2013; Vaisakh and Srinivas, 2011), TS (Abido, 2002), honey bee mating optimization (HBM) (Niknam et al., 2011), and Modified differential evolution (MDE) methods (Sayah and Zehar, 2008). Table 7 and Table 8 show that the best generation cost is obtained by the utilized algorithms. Therefore, the results show the ability of the presented methods to reach the global optimum better than the other optimization approaches.

Table 7. Result comparison for the IEEE 30-bus system

\begin{tabular}{|c|c|c|c|c|}
\hline & EP & DE-OPF & TS & MDE-OPF \\
\hline PG1 (MW) & 173.848 & 176.009 & 176.04 & 175.974 \\
\hline PG2 (MW) & 49.998 & 48.801 & 48.76 & 48.884 \\
\hline PG5 (MW) & 21.386 & 21.334 & 21.56 & 21.51 \\
\hline PG8 (MW) & 22.63 & 22.262 & 22.05 & 22.24 \\
\hline PG11 (MW) & 12.928 & 12.46 & 12.44 & 12.251 \\
\hline PG13 (MW) & 12 & 12 & 12 & 12 \\
\hline Cost (\$/h) & 802.62 & 802.394 & 802.29 & 802.376 \\
\hline & & & & \\
\hline & GA & HBM & GWO & SFLA \\
\hline PG1 (MW) & 175.16 & 178.4646 & 176.8493 & 177.1036 \\
\hline PG2 (MW) & 49.03 & 46.274 & 48.7931 & 48.9199 \\
\hline PG5 (MW) & 19.52 & 21.4596 & 21.5169 & 21.5012 \\
\hline PG8 (MW) & 19.68 & 21.446 & 21.6015 & 21.8577 \\
\hline PG11 (MW) & 17.10 & 13.207 & 11.6872 & 12.1606 \\
\hline PG13 (MW) & 12.00 & 12.0134 & 12.3393 & 11.2553 \\
\hline Cost $(\$ / h)$ & 801.96 & 802.21 & 801.8652 & 801.8280 \\
\hline
\end{tabular}


Table 8. Result comparison for the IEEE 57-bus system

\begin{tabular}{|c|c|}
\hline Method & Fuel cost $(\$ / \mathrm{hr})$ \\
\hline BASE-CASE & 51347.8 \\
\hline GA & 42698 \\
\hline PSO & 42109.7 \\
\hline SFLA & 41872.9 \\
\hline GWO & 41873.188 \\
\hline
\end{tabular}

\section{Conclusions}

This paper proposes the application of two recent evolutionary-based techniques, shuffled frog leaping algorithm and grey wolf optimizer, to solve the optimal power flow problem in electrical power system. These techniques handle the generation fuel cost optimization problem as a single objective function. The performance of the utilized algorithms was tested on the standard IEEE 30-bus and 57-bus test systems and compared with other existing optimization methods from the literature. The results showed that SFLA and GWO produced the lowest fuel cost among the other algorithms under comparison. Moreover, they have similar convergence characteristics where optimal solution is obtained within few number of iterations. However, GWO has only 2 controlling parameters and faster execution time. Thus, based on the performed analysis, GWO is the most promising technique for solving the complicated OPF problem.

\section{Nomenclature}

$\begin{array}{ll}\mathrm{F}_{\mathrm{T}} & \text { Total operating cost of power generation } \\ \mathrm{P}_{\mathrm{i}} & \text { Real power generated } \\ \mathrm{a}_{\mathrm{i}}, \mathrm{b}_{\mathrm{i}}, \mathrm{c}_{\mathrm{i}} & \text { Fuel cost coefficients } \\ \mathrm{NG} & \text { Total number of generator buses } \\ \mathrm{NB} & \text { Total number of buses } \\ \mathrm{NV} & \text { Total number of voltage controlled buses } \\ \mathrm{P}_{\mathrm{i}}, \mathrm{Q}_{\mathrm{i}} & \text { Active and reactive power injection into bus } \mathrm{i} \\ \mathrm{P}_{\mathrm{di}}, \mathrm{Q}_{\mathrm{di}} & \text { Active and reactive load on bus } \mathrm{i} \\ \mathrm{P}_{\mathrm{gi}}, \mathrm{Q}_{\mathrm{gi}} & \text { Active and reactive power generation on bus } \mathrm{i} \\ \mathrm{V}_{\mathrm{i}} & \text { Voltage magnitude on bus } \mathrm{i} \\ \delta_{\mathrm{i}} & \text { Voltage phase angle on bus } \mathrm{i} \\ \mathrm{Y}_{\mathrm{ij}}=\mathrm{G}_{\mathrm{ij}}+\mathrm{j} \mathrm{B}_{\mathrm{ij}} & \text { Admittance matrix elements } \\ X_{i} & \text { Position of the ith frog } \\ P & \text { Number of frogs } \\ \text { rand } & \text { Random value between } 0 \text { and } 1 \\ \mathrm{~N}_{\text {tie }} & \text { Problem dimension } \\ \mathrm{D}_{\text {min }}, \mathrm{D}_{\text {max }} & \text { Minimum and maximum change in the position of frog } \\ \mathrm{D} & \text { Change in frog's position } \\ \vec{A}, \vec{C} & \text { Coefficient vectors } \\ \vec{X} & \text { Prey position vector } \\ \vec{X} & \text { Search agent position vector } \\ \vec{r}_{1}, \vec{r}_{2} & \text { Random vectors from } 0 \text { to } 1 \\ \vec{a} & \text { Vector linearly decreases from } 2 \text { to } 0 \\ X_{\alpha}, X_{\beta}, X_{\delta} & \text { Positions of alpha, beta, and delta wolves } \\ & \end{array}$

\section{References}

Abido, M., 2002, Optimal power flow using tabu search algorithm. Electric Power Components and Systems, Vol. 30, No. 5, pp. 469-483. 
Acharjee, P., 2016, Optimal power flow with UPFC using security constrained self-adaptive differential evolutionary algorithm for restructured power system. International Journal of Electrical Power \& Energy Systems, Vol. 76, 69-81.

Afandi, A. N., \& Miyauchi, H., 2014, Improved artificial bee colony algorithm considering harvest season for computing economic dispatch on power system. IEEJ Transactions on Electrical and Electronic Engineering, Vol. 9, No. 3, pp. 251-257.

Al-Muhawesh, T. A., \& Qamber, I. S., 2008, The established mega watt linear programming-based optimal power flow model applied to the real power 56-bus system in eastern province of Saudi Arabia. Energy, Vol. 33, No. 1, pp. 12-21.

Alguacil, N., \& Conejo, A., 2000, Multiperiod optimal power flow using Benders decomposition. IEEE Transactions on Power Systems, Vol. 15, No. 1, pp. 196-201.

Alsac, O., \& Stott, B., 1974, Optimal load flow with steady-state security. IEEE Transactions on power apparatus and systems Vol. 3, pp. 745-751.

Ambriz-Perez, H., Acha, E., \& Fuerte-Esquivel, C., 2000, Advanced SVC models for Newton-Raphson load flow and Newton optimal power flow studies. IEEE Transactions on Power Systems, Vol. 15, No. 1, pp. 129-136.

Ambriz-Perez, H., Acha, E., Fuerte-Esquivel, C., \& De la Torre, A., 1998, Incorporation of a UPFC model in an optimal power flow using Newton's method. IEE Proceedings-Generation, Transmission and Distribution, Vol. 145, No. 3, pp. 336-344.

Bouzeboudja, H., Chaker, A., Allali, A., \& Naama, B., 2005, Economic dispatch solution using a real-coded genetic algorithm. Acta Electrotechnica et Informatica, Vol. 5, No. 1, p. 4.

Chalermchaiarbha, S., \& Ongsakul, W., 2013, Stochastic weight trade-off particle swarm optimization for nonconvex economic dispatch. Energy Conversion and Management, Vol. 70, pp. 66-75.

Dommel, H. W., \& Tinney, W. F., 1968, Optimal power flow solutions. IEEE Transactions on Power Apparatus and Systems, Vol. 10, pp. 1866-1876.

Duan, Q., Sorooshian, S., \& Gupta, V., 1992, Effective and efficient global optimization for conceptual rainfall runoff models. Water resources research, Vol. 28, No. 4, pp. 1015-1031.

Dubey, H. M., Pandit, M., \& Panigrahi, B. K., 2015, A biologically inspired modified flower pollination algorithm for solving economic dispatch problems in modern power systems. Cognitive Computation, Vol. 7, No. 5, pp. 594-608.

Dubey, H. M., Panigrahi, B. K., \& Pandit, M., 2014, Bio-inspired optimisation for economic load dispatch: a review. International Journal of Bio-Inspired Computation, Vol. 6, No. 1, pp. 7-21.

Eberhart, R., \& Kennedy, J., 1995, A new optimizer using particle swarm theory. Paper Presented at the Micro Machine and Human Science, 1995. MHS'95., Proceedings of the Sixth International Symposium on.

Eusuff, M. M., \& Lansey, K. E., 2003, Optimization of water distribution network design using the shuffled frog leaping algorithm. Journal of Water Resources Planning and Management, Vol. 129, No. 3, pp. 210-225.

Kirschen, D. S., \& Strbac, G., 2004, Fundamentals of Power System Economics: John Wiley \& Sons.

Kumar, S., \& Chaturvedi, D., 2013, Optimal power flow solution using fuzzy evolutionary and swarm optimization. International Journal of Electrical Power \& Energy Systems, Vol. 47, 416-423.

Lin, Q., Huang, G., Bass, B., Huang, Y., \& Liu, L., 2010, The optimization of energy systems under changing policies of greenhouse-gas emission control-A study for the province of Saskatchewan, Canada. Energy Sources, Part A: Recovery, Utilization, and Environmental Effects, Vol. 32, No. 17, pp. 1587-1602.

Mech, L. D., 1999, Alpha status, dominance, and division of labor in wolf packs. Canadian Journal of Zoology, Vol. 77, No. 8, pp. 1196-1203.

Mirjalili, S., Mirjalili, S. M., \& Lewis, A., 2014, Grey wolf optimizer. Advances in Engineering Software, Vol. 69, pp. 46-61.

Momoh, J. A., Adapa, R., \& El-Hawary, M., 1999, A review of selected optimal power flow literature to 1993. I. Nonlinear and quadratic programming approaches. IEEE Transactions on Power Systems, Vol. 14, No. 1, pp. 96-104.

Momoh, J. A., Guo, S., Ogbuobiri, E., \& Adapa, R., 1994, The quadratic interior point method solving power system optimization problems. IEEE Transactions on Power Systems, Vol. 9, No. 3, pp. 1327-1336.

Niknam, T., Narimani, M., Aghaei, J., Tabatabaei, S., \& Nayeripour, M., 2011, Modified honey bee mating optimisation to solve dynamic optimal power flow considering generator constraints, IET Generation, Transmission \& Distribution, Vol. 5, No. 10, pp. 989-1002.

Praveen, J., \& Rao, B. S., 2016, Multi-objective optimization for optimal power flow with IPFC using PSO. Paper Presented at the Electrical Energy Systems (ICEES), 2016 3rd International Conference on.

Sayah, S., \& Zehar, K., 2008, Modified differential evolution algorithm for optimal power flow with non-smooth cost functions. Energy Conversion and Management, Vol. 49, No. 11, pp. 3036-3042.

Senthil, K., \& Manikandan, K., 2010, Economic thermal power dispatch with emission constraint and valve point effect loading using improved tabu search algorithm. International Journal of Computer Applications, Vol. 3, No. 9, pp. 6-11.

Shoults, R. R., \& Sun, D., 1982, Optimal power flow based upon PQ decomposition. IEEE Transactions on Power Apparatus and Systems, No. 2, pp. 397-405.

Suman, M., Rao, M. V. G., Hanumaiah, A., \& Rajesh, K., 2016, Solution of economic load dispatch problem in power system using Lambda Iteration and back propagation neural network methods. International Journal on Electrical Engineering and Informatics, Vol. 8, No. 2, p. 347. 
Toffolo, A., \& Lazzaretto, A., 2002, Evolutionary algorithms for multi-objective energetic and economic optimization in thermal system design. Energy, Vol. 27, No. 6, pp. 549-567.

Tyagi, N., Dubey, H. M., \& Pandit, M., 2016, Economic load dispatch of wind-solar-thermal system using backtracking search algorithm. International Journal of Engineering, Science and Technology, Vol. 8, No. 4, pp. 16-27.

Vaisakh, K., \& Srinivas, L., 2010, A genetic evolving ant direction DE for OPF with non-smooth cost functions and statistical analysis. Energy, Vol. 35, No. 8, pp. 3155-3171.

Vaisakh, K., \& Srinivas, L., 2011, Evolving ant direction differential evolution for OPF with non-smooth cost functions. Engineering Applications of Artificial Intelligence, Vol. 24, No. 3, pp. 426-436.

Wei, H., Sasaki, H., Kubokawa, J., \& Yokoyama, R., 1998, An interior point nonlinear programming for optimal power flow problems with a novel data structure. IEEE Transactions on Power Systems, Vol. 13, No. 3, pp. 870-877.

Yuryevich, J., \& Wong, K. P., 1999, Evolutionary programming based optimal power flow algorithm. IEEE Transactions on Power Systems, Vol. 14, No. 4, pp. 1245-1250.

Zaman, M., Elsayed, S. M., Ray, T., \& Sarker, R. A., 2016, Evolutionary algorithms for dynamic economic dispatch problems. IEEE Transactions on Power Systems, Vol. 31, No. 2, pp. 1486-1495.

Zimmerman, R. D., Murillo-Sánchez, C. E., \& Gan, D. (1997). MATPOWER: A MATLAB power system simulation package. Manual, Power Systems Engineering Research Center, Ithaca NY, 1.

\section{Biographical notes}

Amr Khaled was born in Cairo, Egypt, on September 6, 1989. He received the B.Sc. degree in electrical engineering- Power and machine department in 2011 from Ain shams university, Egypt. He is currently a M.Sc. student at the Department of Engineering Physics and Mathematics, University of Ain shams, Egypt. His areas of research include optimal power flow, Artificial intelligence and optimization techniques.

Ahmed El-Rafei received his Ph.D. degree in computer science in 2012 from the University of Erlangen-Nuremberg, Germany. His Ph.D. thesis was on diffusion tensor image processing and analysis of the visual pathway in glaucoma. Since 2012, Dr. El-Rafei is an assistant professor of engineering mathematics in the faculty of engineering, Ain Shams University, Egypt. His research interests include image processing, medical image analysis, machine learning, and computer science applications in engineering.

Niveen Badra is the Head of the department of Physics and Engineering Mathematics at Faculty of Engineering, Ain Shams University. She is a Professor of Engineering Mathematics since 2008. Her interests are in Optimization and Engineering Applications, Applied Statistics with engineering applications, Numerical techniques with engineering applications, Traffic volume forecasting and Operational management.

Almoataz Y. Abdelaziz received the B.Sc. and M.Sc. degrees in electrical engineering from Ain Shams University, Egypt, in 1985 and 1990 , respectively, and the $\mathrm{Ph} . \mathrm{D}$. degree in electrical engineering according to the channel system between Ain Shams University, Egypt, and Brunel University, U.K., in 1996. He is currently a Professor of electrical power engineering at Ain Shams University. Dr. Abdelaziz is the chair of IEEE Education Society chapter in Egypt, senior editor of Ain Shams Engineering Journal, editor of Electric Power Components \& Systems Journal, editorial board member, associate editor and editorial advisory board member of several international journals and conferences. He is also a member in IET and the Egyptian Sub-Committees of IEC and CIGRE'. He has been awarded many prizes for distinct researches and for international publishing from Ain Shams University, Egypt. He has authored or coauthored more than 300 refereed journal and conference papers in his research areas which include the applications of artificial intelligence, evolutionary and heuristic optimization techniques to power system operation, planning, and control.

Received January 2017

Accepted March 2017

Final acceptance in revised form April 2017 